\title{
Structural differences within the loop E motif imply alternative mechanisms of viroid processing
}

\author{
ROBERT A. OWENS ${ }^{1}$ and TILMAN BAUMSTARK ${ }^{2}$ \\ ${ }^{1}$ Molecular Plant Pathology Laboratory, Beltsville Agricultural Research Center, Beltsville, Maryland 20705, USA \\ ${ }^{2}$ Department of Biological Sciences, University of the Sciences in Philadelphia, Philadelphia, Pennsylvania 19104, USA
}

\begin{abstract}
Viroids replicate via a rolling circle mechanism, and cleavage/ligation requires extensive rearrangement of the highly basepaired native structure. For Potato spindle tuber viroid (PSTVd), the switch from cleavage to ligation is driven by the change from a multibranched tetraloop structure to a loop E conformation. Here we present evidence that processing of Citrus viroid III (CVd-III), a member of a related group of viroids that also replicate in the nucleus, may proceed via a distinct pathway. Chemical probing of PSTVd and CVd-III miniRNAs with DMS and CMCT revealed that the loop E motifs of these two viroids have quite different tertiary structures. As shown by temperature gradient gel electrophoresis, the presence of two likely Watson-Crick GC pairs results in a significant overall stabilization of the CVd-III loop E-like motif. Unlike PSTVd, the upper strand of the CVd-III loop E-like motif cannot fold into a GNRA tetraloop, and comparison of suboptimal structures indicates that the initial cleavage event could occur on the 5' side of the only GU wobble pair in a helix involving a nearby pair of inverted repeats. According to our model, rearrangement of $3^{\prime}$ sequences into a hairpin stem containing an identical arrangement of GC, GU, and CG base pairs and a second cleavage event is followed by formation of loop $E$, which serves to align the $5^{\prime}$ and $3^{\prime}$ termini of the CVd-III monomer prior to ligation. Because ligation would occur within loop E itself, stabilization of this motif may be needed to hold the $5^{\prime}$ and $3^{\prime}$ termini of $\mathrm{CVd}$-III in position for the host ligase.
\end{abstract}

Keywords: Citrus viroid III; cleavage/ligation; loop E; viroid replication; RNA motifs; viroids

\section{INTRODUCTION}

As appreciation of the biological importance of RNA continues to grow, the way in which the structural properties of different RNA molecules determine their functional capabilities is becoming increasingly clear. The complex architecture of folded RNA is shaped by specific tertiary interactions that involve edge-to-edge base-pairing as well as face-to-face stacking interactions. These tertiary interactions are generally mediated by "RNA motifs," ordered arrays of non-Watson-Crick base pairs that shape distinctive foldings in the phosphodiester backbone of the interacting strands, and are easiest to identify when the phylogenetically conserved nucleotides are located in hairpin loops or internal loops flanked by Watson-Crick helices. Nearly a dozen different RNA structural motifs

Reprint requests to: Robert A. Owens, Molecular Plant Pathology Laboratory, Room 118 Building 004, Beltsville Agricultural Research Center, Beltsville, MD 20705, USA; e-mail: robert.a.owens@ars.usda.gov; fax: (301) 504-5449.

Article published online ahead of print. Article and publication date are at http://www.rnajournal.org/cgi/doi/10.1261/rna.452307. are now known, and the list continues to grow (Leontis and Westhof 2003; Hendrix et al. 2005).

The first RNA motif to be described was the so-called loop E motif located in a 14-nucleotide (nt) internal loop that connects two helical regions in Escherichia coli $5 \mathrm{~S}$ rRNA (Fox and Woese 1973). X-ray crystallography (Correll et al. 1997) and NMR analysis (Dallas and Moore 1997) subsequently revealed that loop $\mathrm{E}$ is actually a helical structure containing seven consecutive non-Watson-Crick base pairs. Loop E motifs in ribosomal RNAs often act as recognition sites for RNA-protein interaction and play a crucial role in organizing multihelix junctions (Allison et al. 1991; Leontis and Westhof 1998b). A similar role in RNARNA interaction has been documented for the loop E motif of the hairpin ribozyme (Butcher and Burke 1994). Several loop E motifs share an exceptional susceptibility to interstrand UV cross-linking involving a specific guanosine and uridine residue (Branch et al. 1985).

Loop E motifs have been identified in both hairpin loops (e.g., the $\alpha$-sarcin-ricin loop in 23S rRNA; Endo et al. 1993) and internal loops like those of bacterial 5S rRNA (Leontis and Westhof 1998a) and Potato spindle tuber viroid (PSTVd) (Branch et al. 1985). In bacterial 5S rRNA, the internal loop 
containing the loop E motif is symmetrical, and the bulged nucleotide characteristic of loop E motifs from eukaryotes and one group of the archaea is missing. The minimal ribozyme-substrate complex derived from the hairpin ribozyme found in the negative strand of the Tobacco ringspot virus satellite RNA is composed of two domains, $\mathrm{A}$ and $\mathrm{B}$. The larger B domain contains an asymmetric internal loop that includes many conserved nucleotides and a loop E motif. High-resolution NMR solution (Butcher et al. 1999) and X-ray crystal (Rupert and Ferré-D’Amaré 2001) structures of domain $B$ have been published, and conformational changes in the loop E motif coincidental with domain docking have been shown to be essential for catalysis (Hampel and Burke 2001).

Viroids are the smallest known agents of infectious disease-small (246-401 nt), highly structured, singlestranded, circular RNAs that lack both a protein capsid and detectable mRNA activity (Flores et al. 2005a). The 29 different species of viroids are assigned to one of two taxonomic families based upon differences in the structural and functional properties of their genomes (Flores et al. 2005b). More than 20 years ago, Branch et al. (1985) showed that PSTVd contains a UV-sensitive loop E motif in the central portion of its rod-like native secondary structure. Viroids replicate via a rolling-circle mechanism (Branch and Robertson 1984), and studies of the "processing" of longer-than-unit-length PSTVd RNAs in nuclear extracts have shown that the switch from cleavage to ligation is driven by a change of the central domain from a tetraloop to a loop E conformation (Baumstark and Riesner 1995; Baumstark et al. 1997; Schrader et al. 2003).

In addition to replication, the loop E motif also modulates other aspects of PSTVd interaction with its plant hosts. For example, replacement of the bulged $\mathrm{C}$ residue at position 259 with a $\mathrm{U}$ results in a dramatic increase in the rate of accumulation in tobacco (Wassenegger et al. 1996; Qi and Ding 2002); similarly, a U/A substitution at position 257 affecting a non-Watson-Crick AU pair leads to the appearance of an unusual "flat top" symptom in tomato (Qi and Ding 2003). Although the molecular mechanism(s) responsible for these effects remains to be determined, Zhong et al. (2006) have recently published a detailed model of the tertiary structure of the PSTVd loop E motif, and this model is being used to guide additional mutational analysis.

The family Pospiviroidae contains a total of five genera (Flores et al. 2005a). Like PSTVd and related pospiviroids, members of the other four genera also contain a conserved central region in which a loop E-like motif is flanked by a pair of inverted repeats. Citrus viroid III (CVd-III) is a member of the genus Apscaviroids, and when mutagenesis of its loop E-like motif failed to yield an assortment of variants similar to those obtained from PSTVd (Owens et al. 2006), we decided to examine the structural properties of its loop E motif in greater detail. As described below, our results indicate that sequence changes within loop $\mathrm{E}$ have led to differences in the stability of this important structural motif and, consequently, the cleavage/ligation mechanism by which multimeric replicative intermediates are converted to monomeric viroid progeny.

\section{RESULTS AND DISCUSSION}

\section{Sequence variation with viroid loop E motifs}

Figure 1A shows the computer-generated, lowest-freeenergy secondary structure of CVd-IIIb, one of two predominant variants in the CVd-III quasispecies (Owens et al. 2000). Like the corresponding portion of PSTVd, the central domain of its rod-like native structure contains a loop E-like motif (horizontal bar). Figure 1B compares the sequences of the PSTVd and CVd-IIIb loop E motifs together with their flanking helices. Note that the trans Hoogsteen/Hoogsteen AA pair that follows a bulged C residue positioned in the deep groove in PSTVd has been replaced by a GA pair in CVd-IIIb. Watson-Crick basepairing resumes at varying distances from this purinepurine pair. As shown in Figure 1B, substitutions involving either the bulged $\mathrm{C}$ residue at position 259 or the nearby $\mathrm{U}$ at position 257 have dramatic effects on the biological properties of PSTVd (Wassenegger et al. 1996; Qi and Ding 2003; Qi et al. 2004). When randomization of the corresponding positions in CVd-IIIb resulted only in the reappearance of the wild-type viroid (Owens et al. 2006), we decided to explore the possible structural effects of these sequence differences in greater detail.

\section{UV cross-linking and temperature gradient gel electrophoresis analysis}

More than 20 years ago, Branch et al. (1985) reported that UV irradiation of PSTVd results in formation of an intramolecular cross-link between nucleotides G98 and U260 in the loop E motif. Loop E formation was later shown to be an important step in the switch from cleavage to ligation during PSTVd replication (Baumstark et al. 1997), but direct evidence for the presence of a functional loop $\mathrm{E}$ motif in other pospiviroids (including CVd-IIIb) has been lacking. When preliminary experiments involving precisely full-length CVd-IIIb RNA transcripts failed to yield convincing evidence for UV cross-linking (data not shown), we continued our studies using a series of "miniRNAs" patterned after the PSTVd construct described by Schrader et al. (2003). The structures of four such miniRNAs are shown in Figure 1C. For ease of comparison, inverted repeats in the upper strand that define the boundaries of their central conserved regions and form the stem of secondary hairpin I are underlined.

The $3^{\prime}$ terminus of the longer (i.e., $148 \mathrm{nt}$ ) version of miniPSTVd 1 contains a 17 -nt sequence duplication and differs only slightly from the PSTVd miniRNA originally 


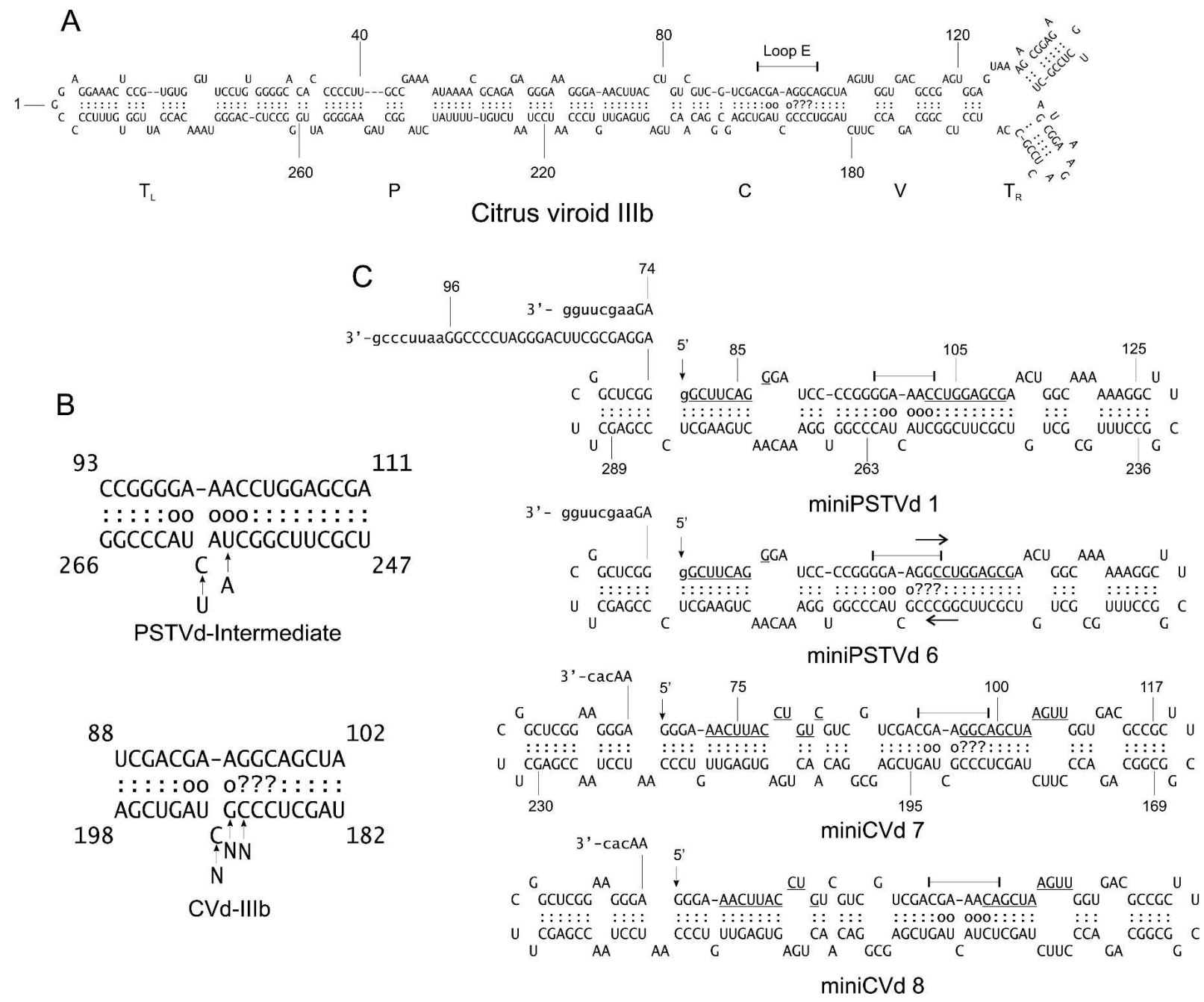

FIGURE 1. Loop E motifs of PSTVd and CVd-III. (A) Lowest-free-energy structure of CVd-IIIb (294 nt). Location of its five structural/function domains and loop E motif (horizontal bar) are shown. (B) Comparison of the loop E motifs of PSTVd and CVd-IIIb. Nucleotide substitutions at PSTVd positions 257 and 259 have dramatic effects on host range and symptom expression; comparable changes in CVd-IIIb rapidly revert to the wild type. (C) Extended secondary structures of two miniPSTVd (above) and two miniCVd (below) RNAs. The inverted repeats marking the boundaries of the upper central conserved region are underlined, and nonviroid nucleotides are shown in lower case. Reference numbers refer to nucleotide positions in the respective full-length circular genomes, and positions of non-Watson-Crick base pairs within the loop E motif are denoted by either open circles (PSTVd) or a combination of open circles and question marks (CVd-III). Note that miniPSTVd 6 and miniCVd 8 contain the loop E motif from CVd-IIIb and PSTVd, respectively; horizontal arrows denote short GC-rich repeats described in the text. Two versions of miniPSTVd 1 and miniPSTVd 6 differing at their $3^{\prime}$ termini as shown at the top of panel $C$ were prepared. The longer versions containing a 17-nt sequence duplication at their 3' termini (Schrader et al. 2003) were used for chemical modification studies; for TGGE comparisons as well as UV cross-linking analysis, the shorter versions with nonduplicated $3^{\prime}$ termini were used.

described by Schrader et al. (2003). A shortened version of miniPSTVd 1 containing only 128 nt lacks this $3^{\prime}$ terminal sequence duplication and was used for temperature gradient gel electrophoresis (TGGE) analysis. To study the effects of individual sequence changes on the susceptibility of the loop E motif to UV cross-linking, we also prepared an additional series of nonduplicated constructs based on miniPSTVd 1. The construct shown (i.e., miniPSTVd 6) contains all five of the sequence changes needed to convert the loop E motif of PSTVd into that of CVd-IIIb. Also shown in Figure 1C are the structures of two miniCVd
RNAs patterned after the shortened version of miniPSTVd 1. The $5^{\prime}$ portions of miniCVd 7 and miniCVd 8 are derived from the central conserved region of CVd-IIIb, and these two RNAs differ only in the sequence of their loop E motifs. miniCVd 7 contains the same loop E-like motif found in full-length, infectious CVd-IIIb; in miniCVd 8, these sequences have been replaced by the corresponding sequences from PSTVd.

Figure 2 presents results from a series of UV crosslinking and TGGE analyses carried out with these miniRNAs. Before analysis, all RNAs were annealed under the 
A

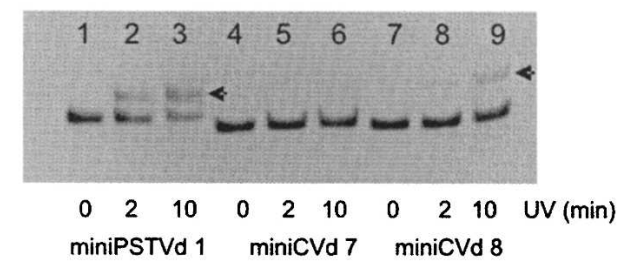

B

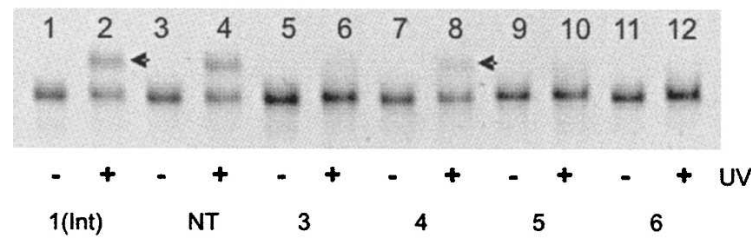

D

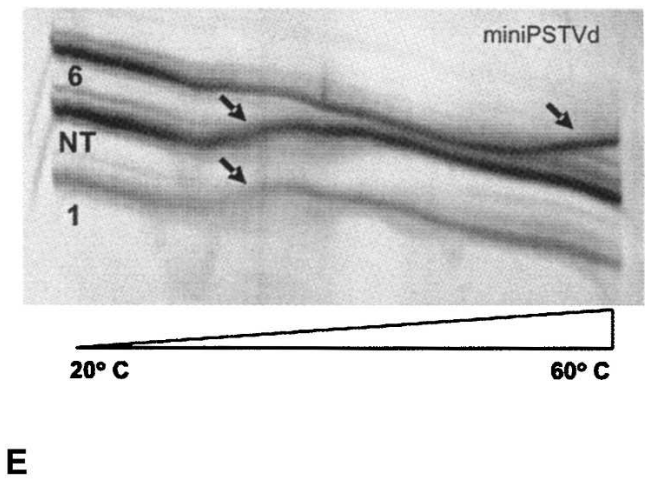

C

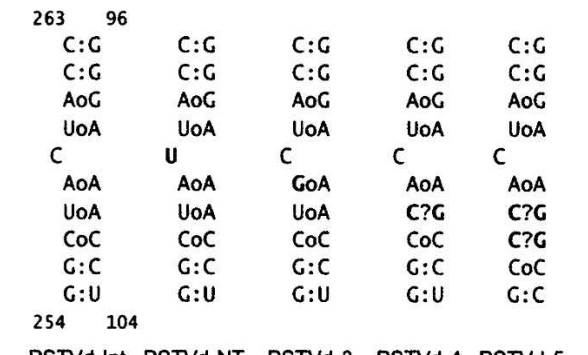

PSTVd-Int PSTVd-NT PSTVd-3 PSTVd-4 PSTVd-5
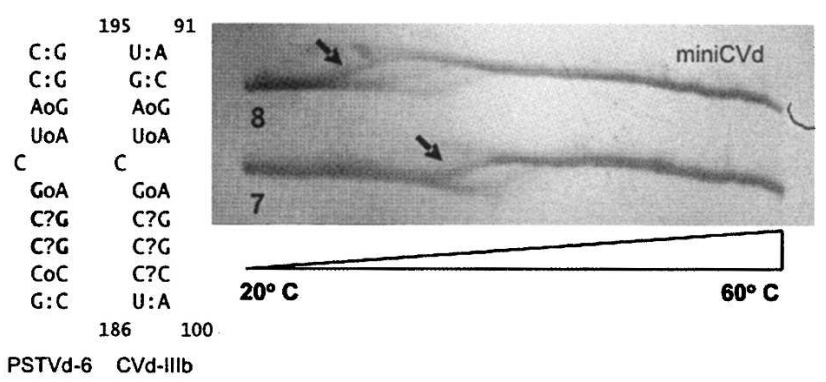

\section{Crosslinking}

FIGURE 2. Structural properties of the PSTVd and CVd-III loop E motifs. Results from UV cross-linking $(A-C)$ and TGGE analysis $(D, E)$ are shown. (A) Relative susceptibility of miniPSTVd 1 (lanes 1-3), miniCVd 7 (lanes 4-6), and miniCVd 8 (lanes 7-9) to UV-induced cross-linking. Filled arrow, position of the slowly migrating lariat structure formed by cross-linking PSTVd positions G98 and U260 or the equivalent positions in CVd-IIIb. $(B, C)$ Effect of selected mutations on the susceptibility of miniPSTVd 1 to UV cross-linking. Locations of mutations are shown in bold; samples in the even-numbered lanes were UV irradiated for 4 min. Note that the loop E motifs of miniPSTVd 6 and miniCVd 7 are identical in sequence. $(D, E)$ Relative stabilities of the PSTVd and CVd-III loop E motifs as measured by TGGE. A temperature gradient of $20^{\circ} \mathrm{C}-60^{\circ} \mathrm{C}$ was applied perpendicular to the direction of electrophoresis in both panels, and major transitions are marked with arrows. Panel $D$ compares the behavior of three miniPSTVd RNAs in the presence of $0.2 \times \mathrm{TBE}$ and $2.5 \mathrm{mM} \mathrm{NaCl}$. Note that replacement of the entire PSTVd loop E motif (no. 6) results in a dramatic shift of the denaturation profile to higher temperatures. Panel $E$ compares the behavior of miniCVd RNAs 7 and 8 in the presence of $0.2 \times \mathrm{TBE}$ alone. Note the presence of multiple transitions (indicating the likelihood of coexisting structures) for both constructs.

high salt-slow cool conditions previously shown, in the case of PSTVd, to favor the formation of an extended conformation containing the loop E motif (Baumstark and Riesner 1995). As shown in Figure 2A, the loop E motif of PSTVd was readily cross-linked by UV irradiation, both in its normal context (i.e., Fig. 2A, miniPSTVd 1, lanes 1-3) or when flanked by helical regions derived from CVd-IIIb (i.e., Fig. 2A, miniCVd 8, lanes 7-9). Also visible was the influence of the length/sequence of the flanking helices on UV sensitivity. The loop E-like motif of CVd-IIIb, in contrast, appeared to be almost totally unaffected by even 10 min of UV irradiation (Fig. 2A, lanes 4-6).

Having shown that the loop E-like motif of CVd-IIIb is resistant to UV cross-linking, we next measured the contributions of individual sequence changes to this behavior. Results are presented in Figure 2B, and Figure 2C compares the structures of all six loop E mutants tested to that of wild-type CVd-IIIb. Comparing lanes 1,2 with lanes 5,6 in
Figure $2 \mathrm{~B}$, it can be clearly seen what was predicted by Leontis et al. (2002) to be an isosteric $A A \rightarrow G A$ substitution involving the trans Hoogsteen-Hoogsteen pair virtually abolished cross-linking. In good agreement with this result, the reciprocal change resulting in an AG pair had also failed to cross-link (Schrader et al. 2003). Although also inhibitory, replacing the adjacent UA pair with a CG pair (Fig. 2B,C, lanes 7,8) had a much smaller effect. Finally, comparison of lanes 3,4 with lanes 1,2 in Figure $2 \mathrm{~B}$ revealed that replacing the bulged $\mathrm{C}$ residue by $\mathrm{U}$ (a change known to have a dramatic effect on host range) had little or no effect on cross-linking.

Although simple and easy to perform, the UV crosslinking assay is quite sensitive to relatively small changes in secondary/tertiary structure. Because cleavage/ligation of pospiviroid replicative intermediates is a dynamic process that involves concerted rearrangement of the entire central conserved region (and not just the loop E motif), we next 
compared the denaturation profiles of selected miniPSTVd and miniCVd RNAs using TGGE. As shown in Figure 2, D and $\mathrm{E}$, these analyses revealed that even small changes in the loop E motif have dramatic effects on the ability of the central conserved region of PSTVd or CVd-III to rearrange. Figure 2D compares the denaturation profiles of three different miniPSTVd RNAs. Two of these molecules (i.e., miniPSTVd 1 and miniPSTVd NT) were efficiently crosslinked by UV irradiation and differ only in the nature of the bulged nucleotide in the loop E motif; in miniPSTVd 6, the entire PSTVd loop E motif has been replaced by the corresponding sequences from CVd-IIIb. Note (1) the nearidentical behavior of miniPSTVd 1 and miniPSTVd NT and (2) the striking change in the overall shape of the transition and $\sim 20^{\circ} \mathrm{C}$ increase in denaturation temperature visible with miniPSTVd 6. Clearly, while certain limited changes in the loop $\mathrm{E}$ motif leave unaltered the ability of the central conserved region to undergo replication-related rearrangements, the effects of more-extensive changes appear to extend into the flanking portions of the central conserved region.

Figure 2E presents results from the reciprocal analysis in which TGGE was used to monitor the effect of replacing the loop E-like motif of CVd-IIIb with the corresponding sequences from PSTVd. Especially noteworthy in this analysis are (1) the presence of multiple independent transitions for both miniCVd 7 and miniCVd 8, and (2) a decrease of $7^{\circ} \mathrm{C}-8^{\circ} \mathrm{C}$ in $\mathrm{T}_{\mathrm{m}}$ values associated with the presence of PSTVd loop E motif. Although one might expect replacement of a single trans Hoogsteen/sugar edge AU pair by two GC pairs of unknown structure to stabilize the loop E motif of CVdIIIb, why both miniRNAs should exhibit multiple transitions was not immediately obvious. One possibility (discussed in more detail below) is that miniCVd RNAs are able to assume several, energetically equivalent conformations under the high salt-slow cool annealing conditions that favor formation of a single extended ExL structure for miniPSTVd (Baumstark and Riesner 1995).

\section{Chemical mapping of PSTVd and CVd-IIIb loop E motifs}

Based on a combination of comparative sequence analysis and NMR and X-ray crystal structures of other loop E motifs, Zhong et al. (2006) have recently proposed that the loop E motif of PSTVd contains (1) an A261/G98 trans Hoogsteen/sugar edge pair, (2) a U260/C259 cis Hoogsteen/sugar edge pair, (3) a U260/A99 trans Watson-Crick/Hoogsteen edge pair, (4) an A258/A100 trans Hoogsteen/Hoogsteen edge pair, (5) an A101/U257 trans Hoogsteen/sugar edge pair, and (6) a C256/C102 cis Watson-Crick/Watson-Crick bifurcated pair. As shown in Figure 1B, the loop E-like motif of CVd-IIIb contains one additional base pair; i.e., the A101/U257 trans Hoogsteen/ sugar edge pair in PSTVd has been replaced by two GC pairs of unknown geometry. In addition, the trans Hoogs-
teen/Hoogsteen edge pair involving PSTVd nucleotides A258 and A100 has been replaced by a GA pair. To assess the effect of these changes on the overall architecture of the loop E motif, we compared the sensitivities of two pairs of miniRNAs to chemical modification.

Initial experiments compared the sensitivity of miniPSTVd RNAs 1 and 6 to modification by dimethylsulfate (DMS) and 1-cyclohexyl-3-(2-morpholinoethyl)-carbodiimide metho-p-toluene sulfonate (CMCT). Incubations were carried out under both "native" and "semi-denaturing" conditions, and as shown in Figure 3A, the modification pattern observed for miniPSTVd 1 was consistent with the tertiary structure proposed by Zhong et al. (2006). In particular, the $\mathrm{N} 1$ positions of four adenosine residues (i.e., A99-A101 and A261) were readily modified by DMS. C259 and A258 reacted less strongly with DMS. Consistent with the expected highly structured local geometry in the loop E motif, CMCT reacted weakly with both U257 and U260. CMCT reacted much more strongly with one of two $\mathrm{U}$ residues in the tetraloop that closes the right arm of the molecule (data not shown).

Examination of a parallel set of reactions containing miniPSTVd 6 revealed a strong CMCT reaction at the position corresponding to U104. As shown in Figure 1C, this position was expected to be part of a helix containing eight Watson-Crick base pairs that adjoins the loop E motif. Close inspection of nearby sequences, however, revealed a pair of short GC-rich direct repeats created by the sequence changes introduced into miniPSTVd 6. A shift of only one base pair between the upper and lower strands is sufficient to align these newly created GGCC sequences, thereby creating a bulge loop containing the CMCT-reactive U104 and, most importantly, partially disrupting the loop E motif. Further evidence for such a shift was provided by the dramatically altered melting profile of miniPSTVd 6 (see Fig. 2D).

To address these problems, a second series of chemical probing experiments was carried out using miniCVd 7 and miniCVd 8, a pair of RNAs showing no evidence of unanticipated structural rearrangement during TGGE analysis (see Fig. 2E). As shown in Figure 3A, the DMS and CMCT modification patterns for miniCVd 8 were quite similar to that seen with miniPSTVd 1; e.g., strong DMS reactions at A99-A101 and A261 and weaker CMCT reactions at U257 and U260. Note, however, that C102 has now become strongly reactive with DMS, possibly as a result of the substitution of a GC base pair for an AU base pair in the flanking helix.

Turning to miniCVd 7, we see further evidence for structural differences between the loop E motifs of CVd-III and PSTVd. Just as in miniCVd 8, residues A94-A95, C98, and A193 in all reacted strongly with DMS. Also reacting strongly with DMS were two additional positions, i.e., C187 and the bulged $C$ at position 191C. Increased accessibility of the bulged $\mathrm{C}$ in miniCVd 7 to DMS modification indicates that the $\mathrm{AA} / \mathrm{GA}$ substitution at the adjacent 
A

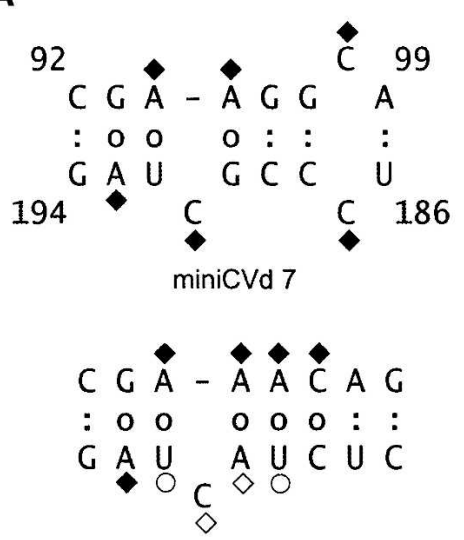

$\operatorname{miniCVd} 8$

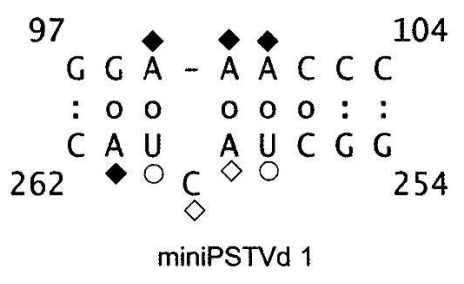

- DMS (strong)

$\diamond$ DMS (weak)
B
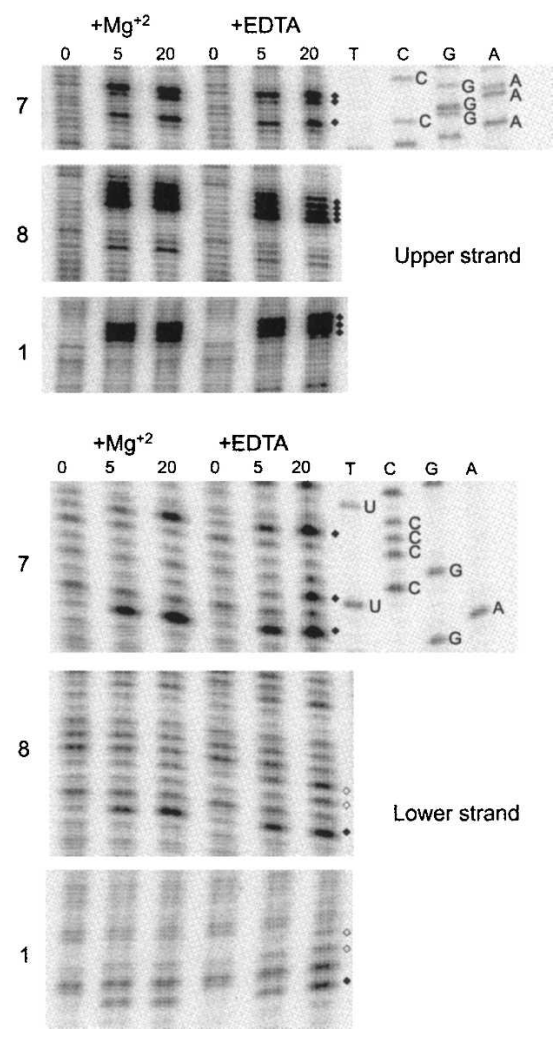

FIGURE 3. Chemical reactivity of individual positions within the loop E motifs of CVd-IIIb and PSTVd. (A) Summary of modification patterns following treatment with DMS (diamonds) and CMCT (circles). Results for three different miniRNAs are shown. Filled symbols indicate strong reactivity and/or reactivity in the presence of $\mathrm{Mg}^{2+} .(B)$ Modification of miniPSTVd 1, miniCVd 7, and miniCVd 8 by DMS. Results obtained under both "native" $\left(+\mathrm{Mg}^{2+}\right.$, medium salt) and semi-denaturing (+EDTA, low salt) conditions are presented, and only residues comprising the loop E motif are shown. Modification reactions were allowed to proceed for 0 , 5, or $20 \mathrm{~min}$. Solid diamonds, positions reacting strongly with DMS; open diamonds, positions that react weakly or only in the presence of EDTA. Sequencing ladders derived from miniCVd 7 are shown on the right.

position is not truly isosteric. According to Zhong et al. (2006), the $\mathrm{Cl}^{\prime}-\mathrm{Cl}^{\prime}$ distances for these two trans Hoogsteen/Hoogsteen pairs differ by $1.2 \AA$; i.e., $12.2 \AA$ (GA) versus $11.0 \AA$ ( $\mathrm{AA})$. An earlier study had estimated the size of the GA pair as $12.9 \AA$ (Leontis et al. 2002). As shown in Figure 2, the presence of this GA pair also inhibited the UV cross-linking of U192 to G93. Consistent with the change in cross-linking, residue U192 has also become completely inaccessible to CMCT. Replacement of the single trans Hoogsteen/sugar edge AU pair in miniCVd 8 with two GC pairs causes residue $\mathrm{C} 187$ in miniCVd 7 to be become accessible to DMS. The failure of DMS to react with either C188 or C189 suggests that pairing may involve the Watson-Crick edge of these two bases.

\section{Proposed mechanism for Apscaviroid processing}

Longer-than-unit-length PSTVd RNA transcripts are correctly processed in a potato nuclear extract only if the central conserved region is able to fold into a multihelix junction containing at least one GNRA tetraloop hairpin (Baumstark and Riesner 1995). The first cleavage occurs within the stem of this GNRA tetraloop, and a local conformational change stabilizes the newly formed $5^{\prime}$ end by converting the tetraloop into a loop E motif. The second cleavage produces a unit-length linear RNA whose $3^{\prime}$ end is base paired (and probably coaxially stacked) in optimum position for ligation to form the mature circular molecule (Baumstark et al. 1997). In CVd-III, the sequence between the inverted repeats defining the boundaries of the central conserved region cannot fold into a GNRA tetraloop; therefore, processing of CVd-III replicative intermediates must proceed via an alternative pathway. The cleavageligation pathway outlined in Figure 4 accommodates the structural differences between the loop $\mathrm{E}$ motifs of CVd-III and PSTVd, thereby illustrating how evolution appears to have found an alternative solution for this key event in viroid-host interaction.

Figure $4 \mathrm{~A}$ compares the initial cleavage events for CVd-III and PSTVd. In PSTVd, formation of the tetraloop stem creates a GG/CU dinucleotide pairing that contains the G-G bond targeted for initial cleavage. Theoretical calculations carried out with a miniCVd RNA whose $5^{\prime}$ terminus starts in the middle of the central conserved region and $3^{\prime}$ end contains a $14-\mathrm{nt}$ duplication of sequences from the $5^{\prime}$ end indicate that CVd-III can readily form other structures that contain an identical dinucleotide pairing. The optimal structure contains an extensive helical pairing between sequences from the $5^{\prime}$ and $3^{\prime}$ ends that is analogous to the trihelical structure described for PSTVd (Steger et al. 1986; Hecker et al. 1986). While such a structure might be too stable for a newly cleaved molecule to rearrange, other suboptimal foldings that maintain only parts of these helices still include the sandwiched GU base pair. In fact, the fifth best suboptimal structure, shown in the bottom half of the dot plot and enlarged on the left with a difference in $\Delta \mathrm{G}$ compared with the optimal structure of only $2.62 \mathrm{kcal} / \mathrm{mol}$, adopts the precise conformation proposed for the first cleavage event. A host nuclease could then recognize this pairing and cleave the G-G phosphodiester bond.

During the second step in our proposed mechanism, the $5^{\prime}$ cleavage fragment dissociates and the $3^{\prime}$ portion of the 
A

First cleavage

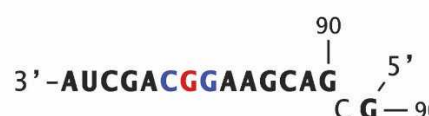

U A

C C
G

C G

G A

$C_{U G} \leftarrow$

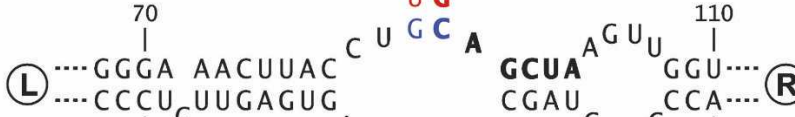

PSTVd

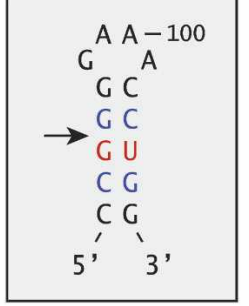

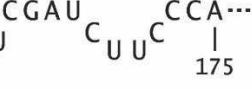

${ }^{U}{ }^{C A}{ }_{A}^{A}$

G

$G$ A

B

Second cleavage

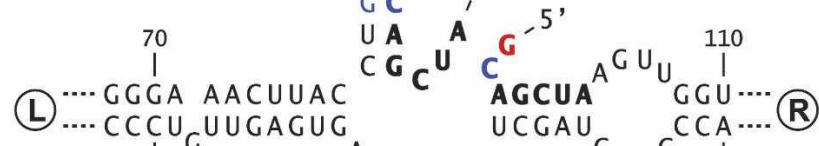

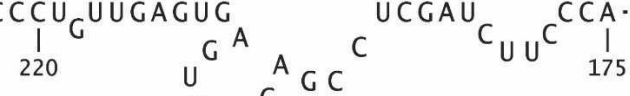

$$
\begin{aligned}
& { }_{C_{A A} C^{C}} C_{C C} C \\
& \text { C G } \\
& \mathrm{G}^{\mathrm{A}} \mathrm{A} \\
& \mathrm{C}_{U} \mathrm{G}
\end{aligned}
$$

mFold dotplot mini-CVdIllb with duplication

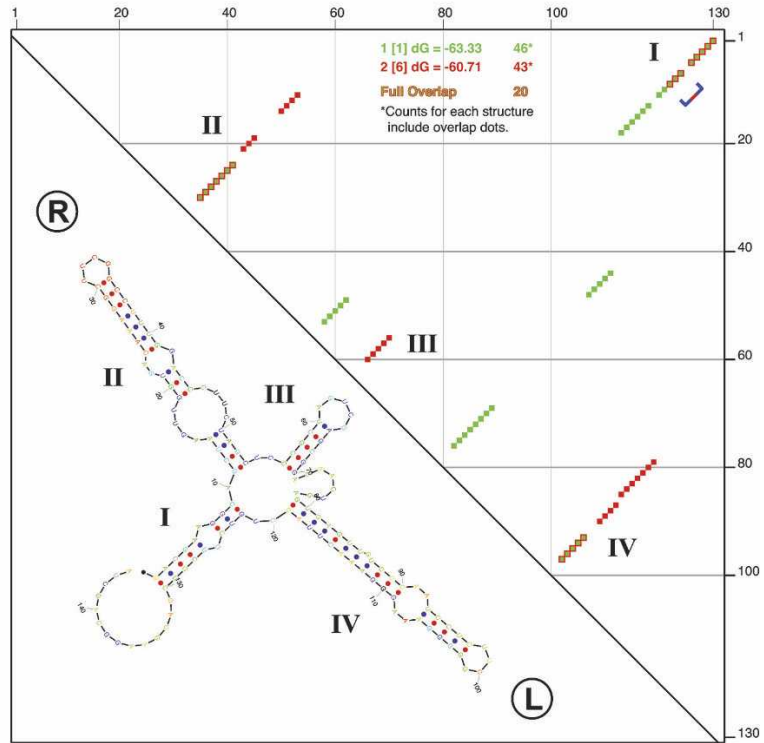

mFold dotplot mini-CVdIIllb post 5 '-cleavage

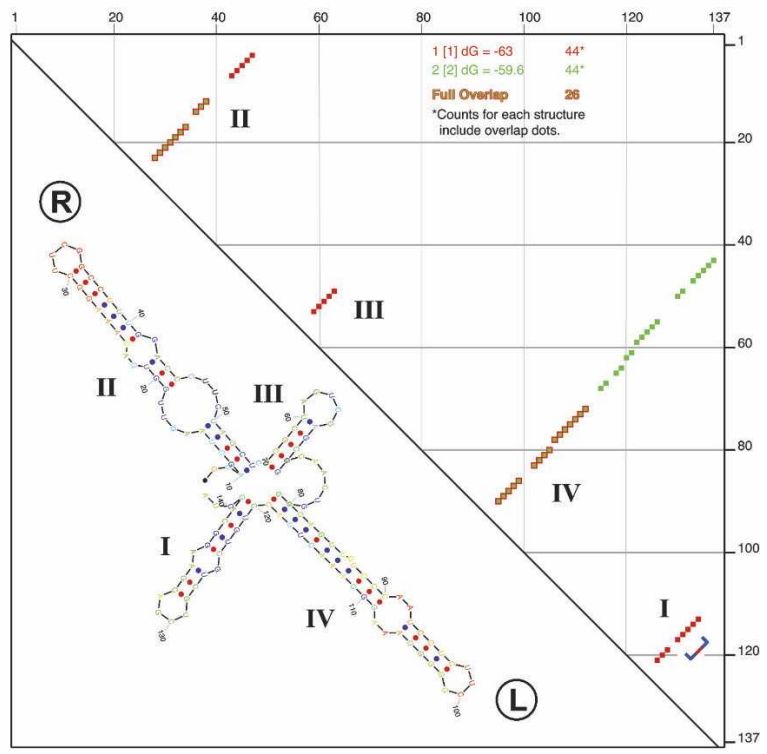

C Ligation

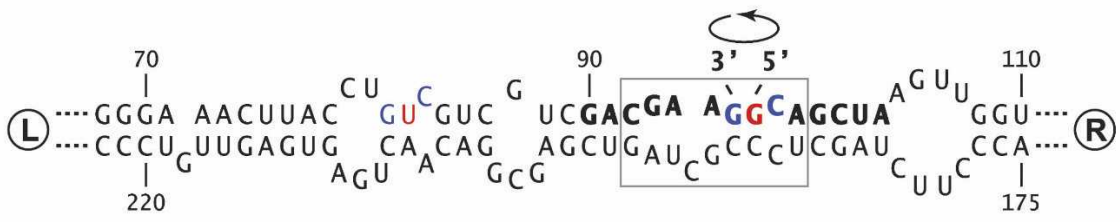

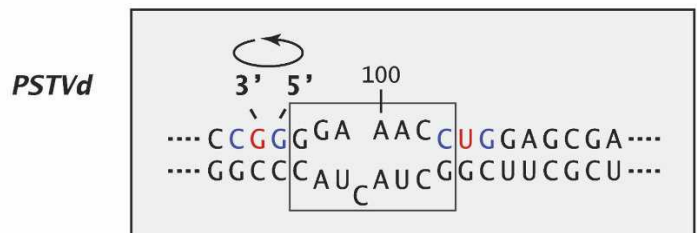

FIGURE 4. (Legend on next page) 
nascent CVd-III RNA refolds into a hairpin stem-loop that pairs the remaining portions of the central conserved region (Fig. 4B). Note that secondary hairpin I reconstitutes the sandwiched GU base pair in an almost identical helical environment for the second cleavage step. Rearrangement to form the processing intermediate shown on the left should occur readily, as indicated by the fact that the conformation containing the second cleavage site is calculated to be the most stable structure. Following the second cleavage and a final rearrangement of the 3' terminus, the $5^{\prime}$ and $3^{\prime}$ termini are then incorporated into the right side of the newly formed loop E-like motif prior to ligation (Fig. 4C). The fact that ligation would occur within the loop E-like motif of CVd-III rather than in the flanking sequences as observed for PSTVd may explain its increased length and stability.

The sequences comprising the loop E-like motif of CVdIIIb are highly conserved among apscaviroids. As shown in Table 1, all eight species contain the same core sequence; i.e., the non-Watson-Crick AG, UA, and GA base pairs surrounding the bulged C residue found in PSTVd, followed by two possible Watson-Crick CG pairs. The first sequence changes appear at the following positions where, in all but one case, a potential Watson-Crick GC or CG pair replaces the symmetrical $\mathrm{C}-\mathrm{C}$ bulge loop found in CVd-IIIb. Changes such as these should have, if any, a stabilizing effect on the alignment of the $5^{\prime}$ and $3^{\prime}$ termini necessary for efficient ligation.

Schuster (2001) has described the many advantages of RNA evolution as a model system in which to study genotype-phenotype relationships. This is especially true for noncoding RNAs such as viroids where evolution is not constrained by the need to maintain coding capacity. The rolling circle nature of viroid replication (Branch and Robertson 1984) creates a need for efficient cleavage/ ligation in order to convert multimeric replicative intermediate to monomeric circular progeny. For the small number of viroids that replicate in the chloroplast, cleavage occurs via hammerhead ribozymes (Côté et al. 2003). For the majority of viroids (including CVd-III) that replicate in the nucleus, cleavage/ligation is catalyzed by host-specified nuclease(s) and requires concerted rearrangement of the central conserved region. Until now, details of this process have been available only for PSTVd (Baumstark and Riesner 1995; Baumstark et al. 1997; Schrader et al. 2003).

We have identified certain differences in stability of the PSTVd and CVd-III loop E motifs as well as the interaction of loop $\mathrm{E}$ with a nearby pair of inverted repeats that strongly suggest that rearrangement of the central conserved region can proceed via more than a single pathway. In contrast to ribosomal RNAs, where loop E motifs appear to organize multihelix loops (Leontis and Westhof 1998b), the central conserved region of CVd-III or PSTVd must "switch" from a multiloop processing structure to an extended structure containing a loop E motif. Zhong et al. (2006) have recently shown how the isostericity matrices developed by Leontis et al. (2002) can be used to rationalize tertiary-structure-function relationships involving the loop E motif of PSTVd and related viroids; furthermore, evidence has also been obtained for the existence of loop $\mathrm{E}$ in vivo (Wang et al. 2007). Our results obtained with a more distantly related viroid indicate that this tertiary-structure-function relationship is yet more complex and contains an important dynamic component. Viroid evolution appears to have identified multiple pathways by which the central conserved region can rearrange to allow efficient cleavage/ligation of RNA replicative intermediates to form mature viroid progeny.

\section{MATERIALS AND METHODS}

\section{Synthesis and purification of miniviroid RNAs}

Plasmid pOS-WT contains a 148-nt cDNA that encodes the central conserved region of PSTVd (intermediate strain) flanked

FIGURE 4. Possible cleavage-ligation mechanism for CVd-III processing. ( $A$, left) Nucleotide changes in the upper strand of CVd-III prevent the loop E sequence from folding into a GNRA tetraloop similar to that observed for PSTVd (inset). In PSTVd, the initial cleavage event (arrow) takes place next to a GU wobble pair sandwiched between GC and CG pairs (highlighted in red and blue). A truncated trihelical folding of CVd-III (see text) contains an almost identical arrangement of base pairs, and the initial cleavage event (arrow) may occur on the $5^{\prime}$ side of its only GU wobble pair. Sequences duplicated between the $5^{\prime}$ and $3^{\prime}$ ends are shown in bold; nucleotide positions are numbered as in previous figures. (Right) Mfold dot plot containing the optimal fold (green) as well as the fifth best suboptimal structure (red) of a miniCVd-III construct with $3^{\prime}$-end duplication as depicted on the left. Base pairs common to both structures are marked by green dots outlined in red. The sandwiched GU base pair is indicated by a red and blue bracket. The suboptimal structure corresponding to the proposed initial processing structure is shown in the bottom triangle. Successive helices constituting the four branches of the structure are designated by roman numerals for better orientation. " $L$ " and " $R$ " denote the tetraloop hairpins, which close the left and right arms of the miniCVd-III construct and have been omitted from the processing structure detail shown on the left. $\left(B\right.$, left) As observed for PSTVd, the first cleavage within the $5^{\prime}$ duplication and dissociation of the upstream sequence then allows the $3^{\prime}$ portion of the CVd-III RNA to rearrange. The resulting hairpin stem contains an identical arrangement of GC, GU, and CG pairs, thereby creating a second potential cleavage site for host nuclease(s). (Right) Mfold dot plot of the miniCVd-III construct from $A$ after the first cleavage and dissociation of the $5^{\prime}$-end fragment. Only the optimal structure (red) and the first suboptimal structure (green) are shown. As depicted in the bottom triangle, the optimal structure corresponds to the rearranged processing intermediate produced by the first cleavage and contains secondary hairpin I at its $3^{\prime}$ terminus. (C) After the second cleavage at the reconstituted $3^{\prime}$-recognition site, final formation of loop E serves to align the new $5^{\prime}$ and $3^{\prime}$ termini of the CVd-III monomer prior to ligation. Note that in contrast to PSTVd (inset), the proposed ligation site (circular arrow) is located within the loop E sequence itself; thus, a more stable loop E-like motif may be needed to hold the $5^{\prime}$ and $3^{\prime}$ termini of CVd-III in position for ligation. 
TABLE 1. Sequence variation within the loop $E$ motif of Apscaviroids

\begin{tabular}{|c|c|c|c|c|c|c|c|c|c|c|}
\hline \multirow[b]{2}{*}{$\begin{array}{l}\text { Reference } \\
\text { sequence } \\
\text { (CVd-IIIb) }\end{array}$} & \multicolumn{10}{|c|}{ Loop E pairings } \\
\hline & $\begin{array}{c}C \\
: \\
\text { G }\end{array}$ & $\begin{array}{l}\mathrm{G} \\
\mathrm{O} \\
\mathrm{A}\end{array}$ & $\begin{array}{l}\mathrm{A} \\
\mathrm{O} \\
\mathrm{U}\end{array}$ & $\mathrm{C}$ & $\begin{array}{l}\text { A } \\
\text { O } \\
\text { G }\end{array}$ & $\begin{array}{l}\text { G } \\
: \\
\text { C }\end{array}$ & $\begin{array}{l}\text { G } \\
: \\
\text { C }\end{array}$ & $\begin{array}{l}\mathrm{C} \\
\mathrm{C}\end{array}$ & $\begin{array}{l}\text { A } \\
: \\
\text { U }\end{array}$ & $\begin{array}{l}\text { G } \\
: \\
\text { C }\end{array}$ \\
\hline CVd-IIIa & & & & & & & & $\begin{array}{c}\text { C } \\
: \\
\text { G }\end{array}$ & C & $\begin{array}{c}\text { A } \\
: \\
\text { U }\end{array}$ \\
\hline AFCVd/AGVd & & & & & & & & $\begin{array}{l}\text { G } \\
: \\
\text { C }\end{array}$ & $\begin{array}{l}U \\
U\end{array}$ & $\begin{array}{l}\mathrm{C} \\
: \\
\mathrm{G}\end{array}$ \\
\hline CBLVd & & & nser & ed $s$ & quen & & & $\begin{array}{c}\text { C } \\
:\end{array}$ & U & C \\
\hline ADFVd & & & & & & & & $\begin{array}{l}\mathrm{G} \\
\mathrm{C}\end{array}$ & $\begin{array}{l}U \\
U\end{array}$ & $\begin{array}{l}\text { U } \\
\text { G }\end{array}$ \\
\hline GYSVd & & & & & & & & $\begin{array}{l}\text { G } \\
\text { G } \\
:\end{array}$ & $\begin{array}{l}\text { U } \\
\text { G } \\
:\end{array}$ & $\begin{array}{l}\mathrm{C} \\
\mathrm{U}\end{array}$ \\
\hline PBCVd & & & & & & & & $\begin{array}{c}\text { U } \\
\text { G } \\
: \\
\text { C }\end{array}$ & $\begin{array}{l}\mathrm{C} \\
\mathrm{U}\end{array}$ & $\begin{array}{c}\mathrm{C} \\
\mathrm{C} \\
: \\
\mathrm{G}\end{array}$ \\
\hline
\end{tabular}

ADFVd indicates Apple dimple fruit viroid; AFCVd, Apple fruit crinkle viroid; AGVd, Australian grapevine viroid; CBLVd, Citrus bent leaf viroid; GYSVd, Grapevine yellow speckle viroid; and PBCVd, Pear blister canker viroid.

by two extra-stable UUCG tetraloops as artificial termini and fused to the promoter sequence for T7 RNA polymerase (Schrader et al. 2003). DNA templates for T7 transcription were synthesized by PCR using a single $5^{\prime}$ primer [5'-CCGAAGCTTAATACGACT CACTAATAG-3'] and one of two $3^{\prime}$ primers; i.e., [5'-CGGA ATTCTCATCATTGTATAGTTC-3'] to maintain the 17-nt terminal duplication encoding PSTVd positions 80-96 or [5'-CCAAG CTTCTCCGAGCCGAAGCT-3'] to produce a shortened, 128-nt RNA transcript lacking this duplication. Sequence changes in the loop E motif of PSTVd were introduced by oligonucleotide overlap mutagenesis (Ho et al. 1989) using primers that contained a minimum of eight perfectly matched nucleotides flanking the targeted position(s).

Using a series of overlapping synthetic oligonucleotides, we also constructed miniCVd 7, a comparable 145-nt nonduplicated cDNA that encodes the central conserved region of CVd-IIIb (Rakowski et al. 1994) flanked by UUCG tetraloops and fused to the promoter sequence for T7 RNA polymerase. After cloning and sequence analysis to verify the absence of unintended mutations, we then converted its loop E motif to that found in PSTVd by oligonucleotide overlap mutagenesis. The resulting construct was designated miniCVd 8. DNA templates for synthesis of miniCVd 7 and miniCVd 8 RNA transcripts were synthesized by PCR as described above using $5^{\prime}$-GTGTTTCCCTTCCGAGCC-3' as the 3' primer.

T7 transcription reactions $(25 \mu \mathrm{L})$ were carried out as suggested by the enzyme supplier (Invitrogen). The resulting miniRNAs were purified by electrophoresis in $6 \%$ polyacrylamide- $0.15 \%$ bisacrylamide gels under denaturing conditions (i.e., $1 \times$ TBE, $8 \mathrm{M}$ urea, $55^{\circ} \mathrm{C}$ ). Full-length miniRNAs were recovered by elution in $0.5 \mathrm{M} \mathrm{NH}_{4}$ acetate- $0.1 \%$ SDS and ethanol precipitation in the presence of a glycogen carrier (Sambrook and Russell 2001). Purified RNAs were dissolved in sterile, RNase-free water and stored at $-20^{\circ} \mathrm{C}$ until use.

\section{UV cross-linking}

Nonduplicated miniPSTVd RNAs were incubated for $45 \mathrm{~min}$ at $40^{\circ} \mathrm{C}$ in the presence of $500 \mathrm{mM} \mathrm{NaCl}-4 \mathrm{M}$ urea- $1 \mathrm{mM} \mathrm{Na}$ cacodylate- $0.1 \mathrm{mM}$ EDTA and then slowly cooled to $20^{\circ} \mathrm{C}$ to favor formation of the extended ExL structure containing loop E (Baumstark and Riesner 1995). Aliquots (5 $\mu \mathrm{L})$ of the annealed RNAs were then placed on parafilm floating on an ice-water bath and irradiated in a UV Stratalinker 1800 (Stratagene) for the times indicated (30 sec-10 min). Following the addition of $15 \mu \mathrm{L}$ of $9 \mathrm{M}$ urea- $0.2 \times \mathrm{TBE}$ containing $0.05 \%$ bromophenol blue and xylene cyanole FF, irradiated samples were fractionated by electrophoresis in $7.5 \%$ polyacrylamide$0.1875 \%$ bisacrylamide gels under denaturing conditions (see above). Cross-link formation was monitored by ethidium bromide staining.

\section{Temperature gradient gel electrophoresis}

Conditions for TGGE analysis were patterned on those used for earlier studies of full-length and longer-than-unit-length PSTVd RNAs (Baumstark and Riesner 1995; Owens et al. 1996). All gels contained $7.5 \%$ polyacrylamide- $0.25 \%$ bisacrylamide, and samples were loaded at 15 -min intervals. Electrophoresis was continued for $45 \mathrm{~min}$ at $500 \mathrm{~V}$ after application of a $20^{\circ} \mathrm{C}-60^{\circ} \mathrm{C}$ temperature gradient. RNAs lacking the 17-nt duplication were annealed under high salt-slow cool conditions described above to favor formation of the ExL structure containing loop $\mathrm{E}$ and then diluted 10 -fold with $10 \%$ glycerol-0.2 $\times$ TBE before application to the gel. Selected miniRNAs were also examined for their ability to assume the multihelix ExM structure. For these analyses, miniRNAs containing the 17-nt duplication were heated for $2 \mathrm{~min}$ at $94^{\circ} \mathrm{C}$ in the presence of $\mathrm{T}_{10} \mathrm{E}_{1}(10 \mathrm{mM}$ Tris- $\mathrm{HCl}-1 \mathrm{mM}$ EDTA at $\mathrm{pH}$ 8.0) and then quickly chilled to $-70^{\circ} \mathrm{C}$ (i.e., low salt-snap cool) before dilution and electrophoresis in the absence of $\mathrm{NaCl}$. Structural transitions were visualized by silver staining (Riesner et al. 1989).

\section{Chemical modification}

After annealing under high salt-slow cool conditions (see above), miniPSTVd and miniCVd RNAs were desalted by passage through AutoSeq G-50 spin columns (GE Healthcare Life Sciences) equilibrated with water. Chemical modification with DMS (positions N1-A, N3-C, N7-G) or CMCT (N3-U, N1-G) was carried out as described by Baumstark and Ahlquist (2001) in the presence of either $5 \mathrm{mM} \mathrm{MgCl}_{2}$ (native conditions) or $1 \mathrm{mM}$ EDTA (semi-denaturing conditions). Primer extension analysis of chemically modified RNAs (Moazed et al. 1986; Ehresman et al. 1987) as well as cycle sequencing of their respective DNA templates was carried out using $\left[5^{\prime}-{ }^{32} \mathrm{P}\right]$-labeled primers $5^{\prime}-$ GGTTCCGGGGATCCCTGAAGCGCTCC-3' (PSTVd) or 5'GTGTTTCCCTTCCGAGCC-3' (CVd-IIIb). 


\section{Structural calculations}

Sequences of PSTVd intermediate strain (359 nt, GenBank NC002030) and CVd-IIIb (294 nt, GenBank S75465) were obtained from the Subviral RNA Database (http://subviral.med. uottawa.ca) containing the complete nucleotide sequences of $>1100$ viroid species and sequence variants. Structural calculations with miniCVd RNAs were carried out using the Mfold server (http://www.bioinfo.rpi.edu/applications/mfold/ rna/form1.cgi) maintained by the Zuker laboratory (Mathews et al. 1999; Zuker 2003).

\section{ACKNOWLEDGMENTS}

We thank Susan M. Thompson for skilled technical assistance and Jon Dinman, Bill Dawson, and Michael Schmitz for critical reading of the manuscript. Financial support to T.B., provided by Reynold Verret, Rod Wigent, and William Law (all at USP), is also greatly appreciated.

Received January 10, 2007; accepted March 10, 2007.

\section{REFERENCES}

Allison, L.A., Romaniuk, P.J., and Bakken, A.H. 1991. RNA-protein interactions of stored 5S RNA with TFIIIA and ribosomal protein L5 during Xenopus oogenesis. Dev. Biol. 144: 129-144.

Baumstark, T. and Ahlquist, P. 2001. The brome mosaic virus RNA3 intergenic replication enhancer folds to mimic a tRNA T $\Psi$ Cstem-loop and is modified in vivo. RNA 7: 1652-1670.

Baumstark, T. and Riesner, D. 1995. Only one of four possible secondary structures of the central conserved region of Potato spindle tuber viroid is a substrate for processing in a potato nuclear extract. Nucleic Acids Res. 2: 4246-4254.

Baumstark, T., Schröder, A.R.W., and Riesner, D. 1997. Viroid processing: switch from cleavage to ligation is driven by a change from a tetraloop to a loop E conformation. EMBO J. 16: 599-610.

Branch, A.D. and Robertson, H.D. 1984. A replication cycle for viroids and other small infectious RNAs. Science 223: 450-455.

Branch, A.D., Benenfeld, B.J., and Robertson, H.D. 1985. Ultraviolet light-induced cross-linking reveals a unique region of local tertiary structure in Potato spindle tuber viroid and HeLa 5S RNA. Proc. Natl. Acad. Sci. 82: 6590-6594.

Butcher, S.E. and Burke, J.M. 1994. A photo-cross-linkable tertiary structure motif found in functionally distinct RNA molecules is essential for catalytic function of the hairpin ribozyme. Biochemistry 33: 992-999.

Butcher, S.E., Allain, F.H.-T., and Feigon, J. 1999. Solution structure of the loop B domain from the hairpin ribozyme. Nat. Struct. Biol. 6: $212-216$.

Correll, C.C., Freeborn, B., Moore, P.B., and Steitz, T.A. 1997. Metals, motif, and recognition in the crystal structure of a 5S rRNA domain. Cell 91: 705-712.

Côté, F., De la Peña, M., Flores, R., and Perreault, J.P. 2003. Ribozyme reactions of viroids. In Viroids (eds. A. Hadidi et al.), pp. 350-356. CSIRO Publishing, Collingwood, Australia.

Dallas, A. and Moore, P.B. 1997. The loop E-Loop D region of Escherichia coli 5S rRNA: The solution structure reveals an unusual loop that may be important for binding ribosomal proteins. Curr. Biol. 5: 1639-1653.

Ehresman, C., Baudin, F., Mougel, M., Romby, P., Ebel, J.-P., and Ehresman, B. 1987. Probing the structure of RNAs in solution. Nucleic Acids Res. 15: 9109-9128.
Endo, Y., Gluck, A., and Wool, I.G. 1993. Ribosomal RNA identity elements for recognition by ricin and by $\alpha$-sarcin: Mutation in the putative CG pair that closes a GAGA tetraloop. Nucleic Acids Symp. Ser. 29: 165-166.

Flores, R., Hernández, C., Martinez de Alba, A.E., Daròs, J.-A., and DiSerio, F. 2005a. Viroids and viroid-host interactions. Annu. Rev. Phytopathol. 43: 4.1-4.23.

Flores, R., Randles, J.W., Owens, R.A., Bar-Joseph, M., and Diener, T.O. 2005b. Viroids. In Virus taxonomy-Eighth Report of the International Committee on Taxonomy of Viruses (eds. C.M. Fauquet et al.), pp. 1145-1159. Elsevier/Academic Press, San Diego, London.

Fox, G.E. and Woese, C.R. 1973. 5S rRNA secondary structure. Nature 256: 505-507.

Hampel, K.J. and Burke, J.M. 2001. A conformational change in the "loop E-like" motif of the hairpin ribozyme is coincidental with domain docking and is essential for catalysis. Biochemistry 40: 3723-3729.

Hecker, R., Wang, Z., Steger, G., and Riesner, D. 1986. Analysis of RNA structures by temperature-gradient gel electrophoresis: Viroid replication and processing. Gene 72: 59-74.

Hendrix, D.K., Brenner, S.E., and Holbrook, S.R. 2005. RNA structural motifs: Building blocks of a modular biomolecule. Q. Rev. Biophys. 38: 221-243.

Ho, S.N., Hunt, H.D., Horton, R.M., Pullen, J.K., and Pease, L.R. 1989. Site-directed mutagenesis by overlap extension using the polymerase chain reaction. Gene 77: 59-74.

Leontis, N.B. and Westhof, E. 1998a. The 5S rRNA loop E: Chemical probing and phylogenetic data versus crystal structure. RNA 4: $1134-1153$.

Leontis, N.B. and Westhof, E. 1998b. A common motif organizes the structure of multi-helix loops in $16 \mathrm{~S}$ and $23 \mathrm{~S}$ ribosomal RNAs. J. Mol. Biol. 283: 571-583.

Leontis, N.B. and Westhof, E. 2003. Analysis of RNA motifs. Curr. Opin. Struct. Biol. 13: 300-308.

Leontis, N.B., Stombaugh, J., and Westhof, E. 2002. The non-WatsonCrick base pairs and their associated isostericity matrices. Nucleic Acids Res. 30: 3497-3531.

Mathews, D.H., Sabina, J., Zuker, M., and Turner, D.H. 1999. Expanded sequence dependence of thermodynamic parameters improves prediction of RNA secondary structure. J. Mol. Biol. 288: 911-940.

Moazed, D., Stern, S., and Noller, H.F. 1986. Rapid chemical probing of conformation in $16 \mathrm{~S}$ ribosomal RNA and $30 \mathrm{~S}$ ribosomal subunits using primer extension. J. Mol. Biol. 187: 399-416.

Owens, R.A., Steger, G., Hu, Y., Fels, A., Hammond, R.W., and Riesner, D. 1996. RNA structural features responsible for Potato spindle tuber viroid pathogenicity. Virology 222: 144158.

Owens, R.A., Yang, G., Gundersen-Rindal, D., Hammond, R.W., Candresse, T., and Bar-Joseph, M. 2000. Both point mutation and RNA recombination contribute to the sequence diversity of Citrus viroid III. Virus Genes 20: 243-252.

Owens, R.A., Thompson, S.M., Sieburth, P.J., and Hilf, M.E. 2006. Mutational analysis of Citrus viroid III symptom expression. In Proceedings 16th Conference IOCV, 2004 (eds. M. Hilf et al.), pp. 280-290. IOCV, Riverside, CA.

Qi, Y. and Ding, B. 2002. Replication of Potato spindle tuber viroid in cultured cells of tobacco and Nicotiana benthamiana: The role of specific nucleotides in determining replication levels for host adaptation. Virology 302: 445-456.

Qi, Y. and Ding, B. 2003. Inhibition of cell growth and shoot development by a specific nucleotide sequence in a noncoding viroid RNA. Plant Cell 15: 1360-1374.

Qi, Y., Pelissier, T., Itaya, A., Hunt, E., Wasseneger, M., and Ding, B. 2004. Direct role of a viroid RNA motif in mediating direction 
RNA trafficking across a specific cellular boundary. Plant Cell 16: $1741-1752$.

Rakowski, A.G., Szychowski, J.A., Avena, Z.S., and Semancik, J.S. 1994. Nucleotide sequence and structural features of the group III citrus viroids. J. Gen. Virol. 75: 3581-3584.

Riesner, D., Steger, G., Zimmat, R., Owens, R.A., Wagenhöfer, M., Hillen, W., Vollbach, S., and Henco, K. 1989. Temperaturegradient gel electrophoresis of nucleic acids: Analysis of conformational transitions, sequence variations, and protein-nucleic acid interactions. Electrophoresis 10: 377-389.

Rupert, P.B. and Ferré-D'-Amaré, A.R. 2001. Crystal structure of a hairpin ribozyme-inhibitor complex with implications for catalysis. Nature 410: 780-786.

Sambrook, J. and Russell, D.W. 2001. Molecular cloning: A laboratory manual, 3rd ed. Cold Spring Harbor Laboratory Press, Cold Spring Harbor, NY.

Schrader, O., Baumstark, T., and Riesner, D. 2003. A miniRNA containing the tetraloop, wobble-pair and loop E motifs of the central conserved region of Potato spindle tuber viroid is processed into a minicircle. Nucleic Acids Res. 31: 988-998.
Schuster, P. 2001. Evolution in silico and in vitro: The RNA model. Biol. Chem. 382: 1301-1314.

Steger, G., Tabler, M., Brüggemann, W., Colpan, M., Klotz, G., Sänger, H.L., and Riesner, D. 1986. Structure of viroid replicative intermediates: physico-chemical studies on SP6 transcripts of cloned oligomeric Potato spindle tuber viroid. Nucleic Acids Res. 14: 9613-9630.

Wang, Y., Zhong, X., Itaya, A., and Ding, B. 2007. Evidence for the in vivo existence of loop E motif of Potato spindle tube viroid. J. Virol. 81: 2074-2077.

Wassenegger, M., Spieker, R.L., Thalmeir, S., Riedel, L., and Sänger, H.L. 1996. A single nucleotide substitution converts Potato spindle tuber viroid (PSTVd) from a noninfectious to an infectious RNA for Nicotiana tabacum. Virology 226: 191-197.

Zhong, X., Leontis, N., Qian, S., Itaya, A., Qi, Y., Boris-Lawrie, K., and Ding, B. 2006. Tertiary structural and functional analyses of a viroid RNA motif by isostericity matrix and mutagenesis reveal its essential role in replication. J. Virol. 80: 8566-8581.

Zuker, M. 2003. Mfold web server for nucleic acid folding and hybridization prediction. Nucleic Acids Res. 31: 3406-3415. 

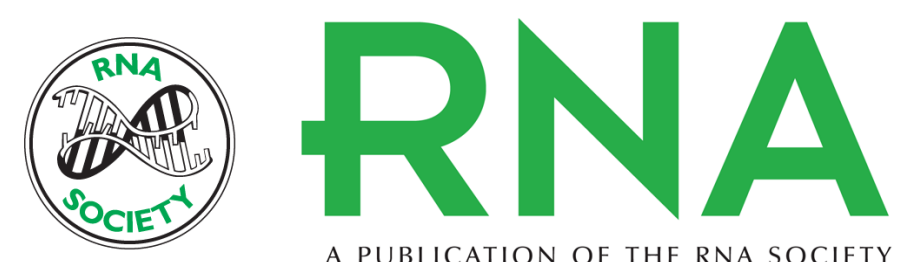

A PUBLICATION OF THE RNA SOCIETY

\section{Structural differences within the loop E motif imply alternative mechanisms of viroid processing}

Robert A. Owens and Tilman Baumstark

RNA 2007 13: 824-834 originally published online April 16, 2007

Access the most recent version at doi:10.1261/rna.452307

References This article cites 39 articles, 9 of which can be accessed free at:

http://rnajournal.cshlp.org/content/13/6/824.full.html\#ref-list-1

\section{License}

Email Alerting Service right corner of the article or click here. 